\title{
Impact of chronic heart failure on adipose tissue functional plasticity - a role for fatty acids?
}

\author{
Andrew D Yale ${ }^{1}$, Malcolm Cobb ${ }^{1}$, Alexander Lyon ${ }^{2}$, Alison Mostyn ${ }^{1}$
}

UNITED KINGDOM $\cdot$ CHINA $\cdot$ MALAYSIA

${ }^{1}$ Faculty of Medicine and Health Sciences, School of Veterinary Medicine and Science, Sutton Bonington Campus, University of Nottingham, LE12 5RD, ${ }^{2}$ NIHR Cardiovascular Biomedical Research Unit, Royal Brompton Hospital

\section{Introduction}

Chronic heart failure (HF) is a major cause of morbidity and mortality in humans and domestic animals. HF is a systemic condition characterised by chronic inflammation and functional changes in non-cardiovascular tissues; obesity is also associated with low level chronic inflammation, with adipose tissue (AT) exhibiting infiltration of macrophages and alterations in fatty acid (FA) profiles $^{(1)}$.

Our hypothesis was that AT from animals with HF will display greater expression of inflammatory mediators and a fatty acid profile consistent with an inflammatory state.

Therefore the aim of this project was to investigate expression of regulators of lipid metabolism and inflammation and fatty acid profiles in AT from a rodent model of HF.

\section{Methods}

Rats with HF post myocardial infarction were compared with age matched non-failing controls. Intra-abdominal (IA) and subcutaneous (SC) adipose tissue depots were sampled after humane euthanasia, and then processed for analysis.

Adult male Sprague-Dawley rats $(\mathrm{N}=6)$ underwent proximal coronary ligation to induce chronic myocardial infarction. Sham ligation was used as control $(\mathrm{N}=6)$. Sixteen weeks later assessment of cardiac function completed and rat humanely euthanased and adipose tissue removed and stored at $-80^{\circ} \mathrm{C}$.

RNA extracted, reverse transcribed and subjected to QPCR. Rat specific primers for several inflammatory mediators and metabolic markers included stearoyl-CoA desaturase-1 (SCD1), interleukin 1 (IL1) and phosphoenolpyruvate carboxykinase (PCK1)

Lipid extracted (Folch method( ${ }^{(2)}$ ); triglycerides (TAG) measured colourometrically, fatty acid profiles assessed using gas chromatography.

Statistical significance was assessed using ANOVA with post hoc Tukey tests

\section{Results}

Triglycerides - Greater storage of TAG observed in HF intraabdominal AT.

Gene Expression - Reduced expression of SCD1 (enzyme that catalyses desaturation to mono-unsaturates) in HF intra-abdominal AT.

Fatty Acids - FA analysis identified differences in several species; including reductions in $\mathrm{C} 10, \mathrm{C} 15$ and $\mathrm{C} 17$ in HF-SC. However, most interesting was a reduction of both $\mathrm{C} 20: 5 \mathrm{n} 3$ (EPA) and C22:6n3 (DHA) in HF-SC.

Figure 1: Confirmation of heart failure in rats $(\mathrm{HF})^{(3)}$
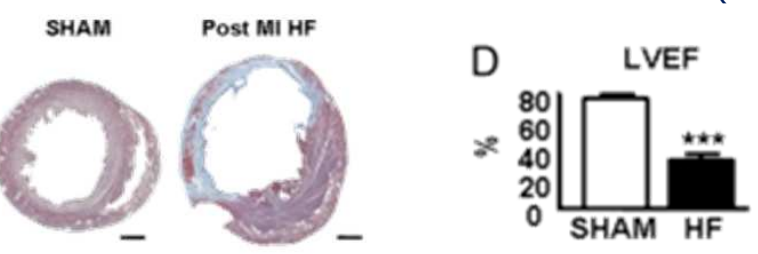

Figure 2: Selected fatty acid profiles in adipose tissue
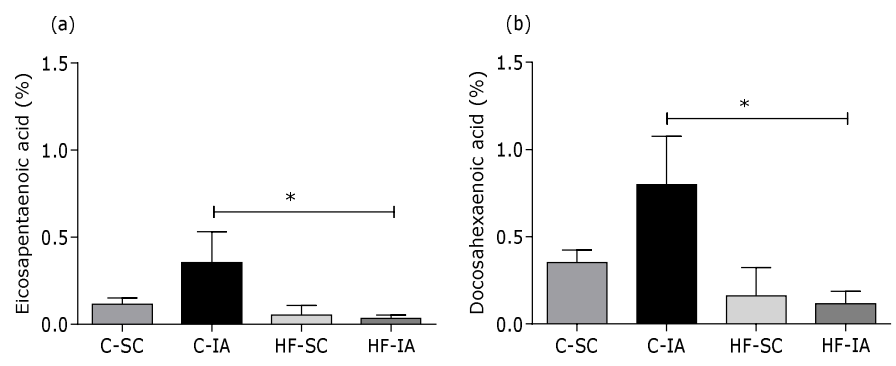

Figure 3: SCD1 expression in adipose tissue

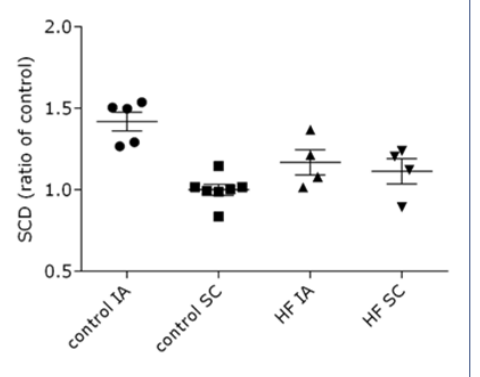

Figure 4: Adipose tissue TAG content

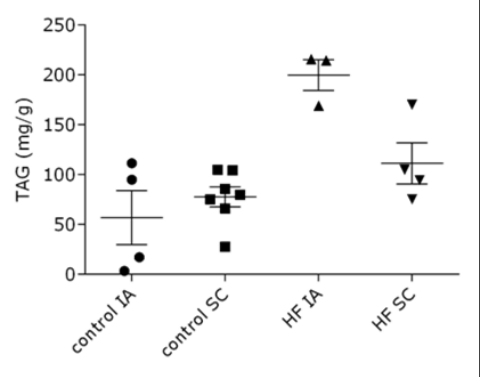

Figure 5: Metabolic pathway for Omega-3 fatty acids

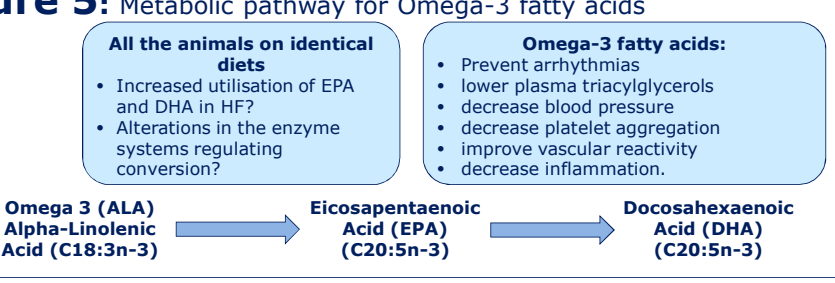

\section{Conclusions}

Despite a reduction in SCD1 expression, we did not detect any difference in mono-unsaturated FA species in the HF rats.

EPA and DHA are omega 3 FAs with recognised antiinflammatory and cardio-protective effects; a reduction in HF suggests altered uptake and metabolism in these animals.

\section{Future Research}

Possible areas for further research are:

- To investigate the impact of SERCA2 gene therapy and whether it can ameliorate the effects of HF on adipose tissue.

- To assess the impact of HF on brown adipose depots given. the emerging roles of BAT in adult mammals.

- To determine whether the FAs act as biomarkers of HF.

Nutritional intervention

\section{Reference}

(1) Cinti S, et al. J Lipid Res. 2005;46.

(2) Folch, J., M. Lees and G. H. Sloane Stanley (1957). "A simple method for the isolation and purification of total lipids from animal tissues." ] Biol

Chem 226(1): 497-509

(3) Lyon, A. R., et al. (2009) Proceedings of the National Academy of Sciences 106(16): 6854-6859.

\section{Acknowledgements}

Financial support was provided by:
Contact

For more information please contact: svyay@nottingham.ac.uk 